Elsevier required licence: (c) <2018>. This manuscript version is made available under the CC-BY-NC-ND 4.0 license http://creativecommons.org/licenses/by-nc-nd/4.0/

The definitive publisher version is available online at $h$ ttps://doi.org/10.1016/j.apenergy.2017.12.042 


\title{
An impact-based broadband aeroelastic energy harvester for concurrent wind and base vibration energy harvesting
}

\author{
Liya Zhao ${ }^{\mathrm{a}}$ and Yaowen Yang ${ }^{\mathrm{b} *}$ \\ ${ }^{a}$ University of Technology Sydney, NSW 2007, Australia \\ ${ }^{b}$ Nanyang Technological University, 50 Nanyang Avenue, Singapore 639798, Singapore \\ *cywyang@ntu.edu.sg
}

\begin{abstract}
This paper proposes a novel broadband energy harvester to concurrently harvest energy from base vibrations and wind flows by utilizing a mechanical stopper. A problem for a conventional wind energy harvester is that it can only effectively harness energy from two types of excitations around its resonance frequency. The proposed design consists of a D-shape-sectioned bluff body attached to a piezoelectric cantilever, and a mechanical stopper fixed at the bottom of the cantilever which introduces piecewise linearity through its impact with the bluff body. The quasi-periodic oscillations are converted to periodic vibration due to the introduction of the mechanical stopper, which forces the two excitation frequencies to lock into each other. Broadened bandwidth for effective concurrent energy harvesting is thus achieved, and at the same time, the beam deflection is slightly mitigated and fully utilized for power conversion. The experiment shows that with the stopper-bluff body distance of $19.5 \mathrm{~mm}$, the output power from the proposed harvesting device increases steadily from $3.0 \mathrm{~mW}$ at $17.3 \mathrm{~Hz}$ to $3.8 \mathrm{~mW}$ at $19.1 \mathrm{~Hz}$ at a wind speed of $5.5 \mathrm{~m} / \mathrm{s}$ and a base acceleration of $0.5 \mathrm{~g}$. A guideline for the stopper configuration is also provided for performance enhancement of the broadband concurrent wind and vibration energy harvester.
\end{abstract}

\section{Keywords}

broadband concurrent energy harvesting; wind energy; vibration; piezoelectric; mechanical stopper; piecewise-linearity

\section{Introduction}

The field of energy harvesting has received ever growing research interests in the recent years. The ultimate goal is to implement self-powered microelectronic systems such as wireless sensor networks and communication devices by eliminating the dependency of batteries, which are of limited lifespans thus require cumbersome replacements. Available energy sources surrounding the electronic systems include solar energy, mechanical vibrations, electromagnetic radiation, thermal gradients and wind flows. These energy sources can be harnessed and converted into electricity as a substitute or backup power supply. For example, researchers have recently studied energy harvesting from mechanical vibrations for applications in rail tracks or roadways (Zhang et al., 2016; Zhang et al., 2017c; Jung et al., 2017).

In the past years, considerable research efforts have been devoted to piezoelectric energy harvesting from base vibrations (Harne and Wang, 2013; Daqaq et al., 2014). A major challenge for traditional linear resonant harvesters is that when the excitation frequency slightly deviates 
from the resonance, a dramatic decrease in the power generation efficiency occurs. Various techniques have been proposed in order to broaden the operational frequency bandwidth and improve the energy conversion efficiency of base vibration energy harvesters. These efforts include developing energy harvesters with close multiple modes (Tang and Yang 2012a; Zhou et al., 2011), introducing nonlinearity by adding magnets to achieve monostable, bistable or tristable responses (Vocca et al., 2012; Tang and Yang 2012b; Zhou et al., 2013, 2014; Harne and Wang, 2013; Daqaq et al., 2014; Yang and Zu, 2016; Yang and Towfighian, 2017), employing frequency up-conversion technique (Gu and Livermore, 2011; Kim et al., 2014; Fu and Yeatman, 2017), etc. Zhou et al. (2013) theoretically and experimentally investigated a tristable piezoelectric energy harvester, which was shown to pass easily the potential wells to achieve wide bandwidth with high energy output compared to a bistable energy harvester with a deeper potential well. Fu and Yeatman (2017) recently reported a methodology to harness low frequency rotational energy by utilizing the frequency up-conversion technique, which was achieved by the magnetic plucking between the tip magnet at the piezoelectric tip and the rotating magnet on a revolving host. Besides the above techniques, impact-based energy harvesters have also been studied by employing mechanical stoppers to achieve piecewise-linearity with bilinear stiffness which extends the bandwidth over resonance (Soliman et al., 2008; Liu et al., 2011, 2012; Wu et al., 2014; Wang et al., 2017). Soliman et al. (2008) proposed the first study on an electromagnetic energy harvester with piecewise-linear restoring force by employing a mechanical stopper. A broadened upsweep bandwidth which was $240 \%$ wider than that of the linear counterpart was experimentally demonstrated. Liu et al. $(2011,2012)$ reported broadband MEMS piezoelectric energy harvesters using both one-sided and two-sided mechanical stoppers. Two mechanical stoppers were employed as synchronous mechanical switches of the optimized synchronous electric charge extraction circuit for a piezoelectric energy harvester in the work of Wu et al. (2014). Recently, Wang et al. (2017) reported a compact piezoelectric vibration energy harvester with tunable resonance and broadened bandwidth by integrating a suspended piezoelectric spring-plate with a top stopper-plate, which achieved multiple nonlinear effects such as duffing-spring effect, impact effect, preload effect, and air elastic effect.

Besides the pre-existing mechanical vibrations, the bulky kinetic energy in the ambient wind flows provides an alternative on-site power source (Morbiato et al., 2014). When a properly supported structure is subjected to wind flows, aeroelastic instabilities will give rise to large amplitude limit cycle oscillations, and the vibration energy can be further converted into electricity via specific electromechanical transduction mechanisms such as piezoelectric effect. Researchers have employed various aeroelastic instabilities to harness the kinetic energy in wind flows, including vortex-induced vibration (VIV) (Akaydin et al., 2012; Goushcha et al., 2014; Dai et al., 2016; Zhang et al., 2017a, 2017b), galloping (Sirohi and Mahadik, 2012; Ewere et al., 2014; Zhao et al., 2013, 2014; Vicente-Ludlam et al., 2015; Dai et al., 2015; Zhao and Yang, 2015), aeroelastic flutter (Bryant and Garcia, 2011; Aquino et al., 2017; Orrego et al., 2017; Wu et al., 2017), etc. Using a VIV-based energy harvester with a piezoelectric cantilevered cylinder, Akaydin et al. (2012) obtained a peak power of around $0.1 \mathrm{~mW}$ at a wind speed of $1.192 \mathrm{~m} / \mathrm{s}$. The driving mechanisms of VIV energy harvesting was subsequently investigated by Goushcha et al. (2014) using particle image velocimetry. Galloping occurs to flexibly supported bluff bodies with certain cross-section geometries of which the aerodynamic coefficients satisfy the Den Hartog criterion (Den Hartog, 1956). With a galloping piezoelectric energy harvester with a square-sectioned bluff body, a peak power of $8.4 \mathrm{~mW}$ was achieved at a wind speed of $8 \mathrm{~m} / \mathrm{s}$ by Zhao et al. (2013). Modal 
convergence flutter energy harvesters using a cantilevered airfoil with coupled torsion and bending motions were studied by Bryant and Garcia (2011) and Wu et al., (2017). Other types of flutter energy harvesters were also reported, such as the flutter energy harvester with an inverted piezoelectric flag recently proposed by Orrego et al. (2017), and the electromagnetic energy harvester exploiting the cross flow flutter of a flexible belt proposed by Aquino et al. (2017). Studies on energy harvesting based on wake galloping with paralleled cylinders (Abdelkefi et al., 2013) and turbulence-induced vibrations with piezoelectric grass (Hobeck and Inman, 2014) were also reported. Moreover, efforts have been devoted to enhancing the wind energy harvesting performance from the mechanical aspect with modified structural configurations (Zhao et al., 2014; Zhao and Yang, 2015; Vicente-Ludlam et al., 2015) and the circuit aspect with optimized power extraction interface (Zhao et al., 2016, 2017; Zhao and Yang, 2017). For example, Zhao and Yang (2015) proposed an effective method for aeroelastic energy harvesting enhancement by adding a beam stiffener as an electromechanical coupling amplifier, which was demonstrated to boost the power generation for all three types of energy harvesters based on galloping, VIV and airfoil flutter. Enhanced galloping energy harvesting was also investigated by Zhao et al. (2016) using a synchronized charge extraction interface and by Zhao et al. (2017) employing a synchronized switching harvesting on inductor interface. Other developments in small-scale wind energy harvesting are available in the recent review works (Young et al., 2014; McCarthy et al., 2016; Zhao and Yang, 2017). Recently, triboelectric nanogenerators as a new group of energy harvesters have been enthusiastically studied for harnessing ambient mechanical energy from vibrations or airflows based on the coupling of triboelectrification and electrostatic induction. Triboelectric charges and potential differences are induced during the periodic physical contact and separation between two materials with distinct electron affinity. It has been shown to be a cost-effective and robust technique for energy harvesting. Interested readers are referred to the work of Zhu et al. (2015), Ahmed et al. (2017), Li et al. (2017) and Phan et al. (2017) for more details.

However, all the above mentioned studies on kinetic energy harvesting have considered only one type of energy source, either pre-existing base vibrations or wind flows. There are many circumstances where wind flows and base vibrations are coexisting, such as on the heavily travelled bridges, subway tunnels, ships, aircrafts, supporting structures of offshore infrastructures, and numerous buoys in the ocean. These two types of energy sources can be simultaneously harvested to power the sensors or other microelectronic de vices. Recently, some researchers have investigated concurrent wind and vibration energy harvesting with an aeroelastic energy harvester (Bibo and Daqaq, 2013a, 2013b; Dai et al., 2014; Yan et al., 2014; Bibo et al., 2015). It was found by Bibo and Daqaq (2013a, 2013b) that for a flutter energy harvester under combined aerodynamic and base vibratory excitations, when the wind speed was below the flutter speed, the flow amplified the output power from base excitations, while beyond the flutter speed, enhanced power generation was achieved for base excitation frequencies very close to the resonance. Similar phenomenon was also observed for a VIV energy harvester (Dai et al., 2014) and for a galloping energy harvester (Yan et al., 2014; Bibo et al., 2015) under concurrent wind flows and base vibrations. However, a major problem with these traditional aeroelastic energy harvesters is that they can effectively harness energy from the combined excitations only around the harvesters' fundamental frequencies. There is only a narrow bandwidth around the resonance where the two energy sources can supplement each other. This is due to coexistence of two different frequencies resulting from the two types of excitations, making the harvester undergo 
quasi-periodic oscillations if the base vibration frequency deviates from the resonance. As a result, the peak displacement amplitude is high in a very wide frequency range, yet the effectively harvested average power is low except around the resonance. Nevertheless, while various techniques have been proposed to enhance energy harvesting from pure base vibrations and pure wind flows, performance enhancement of concurrent base vibration and wind energy harvesting has received far less attention. A series of complex mutual coupling behaviors exist in the traditional linear aeroelastic energy harvester, that is, the aeroelectromechanical coupling between the flow, structure, piezoelectric transducer and electric components. Under combined loadings, the responses are further complicated by the interaction between the coexisting base excitation frequency and aerodynamic forcing frequency. Introducing the broadband techniques for base vibration energy harvesting into the concurrent energy harvesting system will bring more complex coupling behaviors and interactions. Therefore, although broadband concurrent base vibration and wind energy harvesting is highly demanded, there is very little effort devoted into this issue.

To fill this gap, in this paper, we propose a novel design of energy harvester to concurrently harvest energy from base vibrations and wind flows with a broadened bandwidth. The device is designed and fabricated by adding a mechanical stopper to a linear galloping piezoelectric energy harvesting system. The proposed system offers several advantages. The quasi-periodic oscillations are converted to periodic vibration by the introduced mechanical stopper which forces the two excitation frequencies from wind and base vibration to lock into each other. Broadened bandwidth for effective concurrent energy harvesting is thus achieved. At the same time, the peak beam deflections are slightly mitigated and fully utilized for power conversion. The proposed design of integrating an aeroelastic energy harvester with a mechanical stopper has been shown to be a viable solution for broadband concurrent wind and base vibration energy harvesting. To our best knowledge, this is the first study that proposes and experimentally validates the feasibility of broadband concurrent energy harvesting integrating a mechanical stopper.

\section{Device Configuration}

The configuration of the proposed broadband energy harvester for concurrent base vibration and wind energy harvesting is shown in Fig. 1. Galloping instability is employed here due to its capability of oscillating in an infinite wind speed range and large amplitude during limit cycle oscillation. A piezoelectric element is bonded to a cantilever near its fixed end. A D-shapesectioned bluff body is attached to the free end of the cantilever to achieve the wind-induced galloping instability. The flat surface is adjusted to be facing the wind flow at rest which is the most unstable position to give rise to oscillation. Another cantilever is fixed at a certain distance below the cantilever as a mechanical stopper. An energy harvesting interface circuit is connected across the electrodes of the piezoelectric element to further transfer the generated electrical charge, which is resulted from the piezoelectric effect during the alternating deformation of the energy harvester.

The resonance frequency of the stopper is chosen to be much higher than the energy harvester. When the oscillation amplitude of the energy harvester is sufficiently high, the bluff body will impact with the stopper, resulting in a sudden increase in the effective stiffness (Soliman et al., 2008; Liu et al., 2011, 2012). Such piecewise linear stiffness brings an extension of the resonance to a wider range of frequencies, within which the harvester can effectively harness the concurrent energy from both base vibration and wind flow. The stopper cantilever will oscillate at its own 
high resonance frequency when the bluff body departs from the contact in each cycle, achieving a frequency up-conversion. If a piezoelectric element is bonded to the root area of the stopper, this part of strain energy can be further harnessed and transferred to electricity. In this study, we focus on the power generation performance of the galloping energy harvester without adding piezoelectric materials on the stopper.
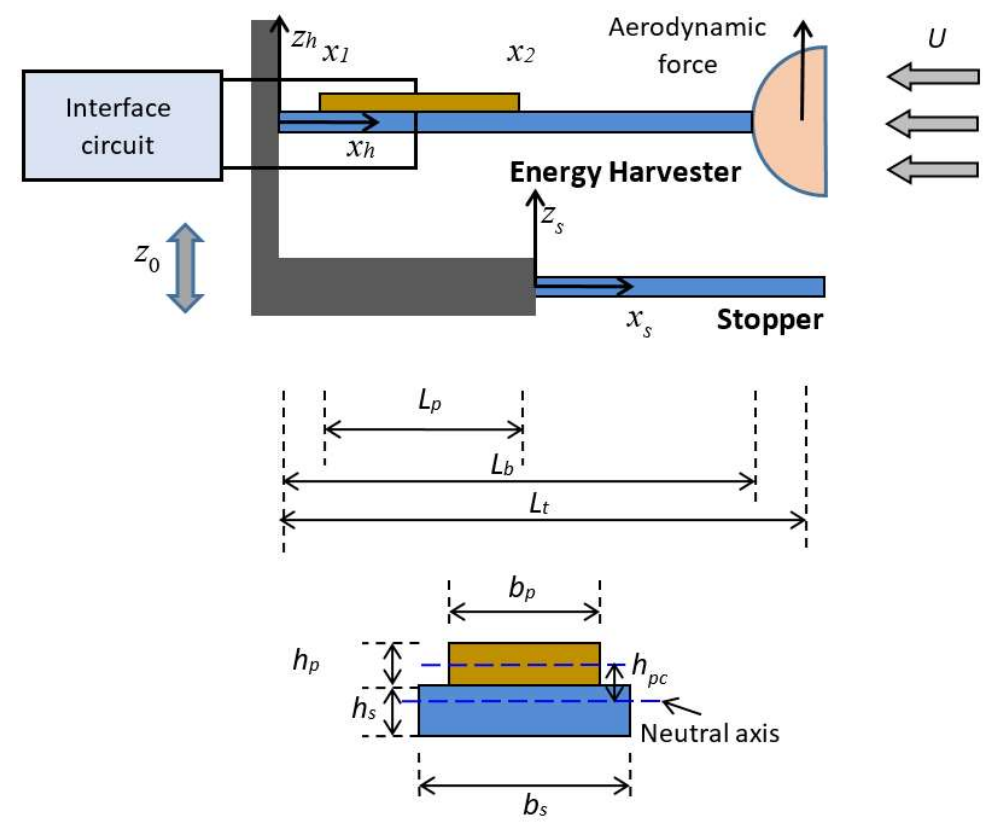

Fig. 1 Configuration of proposed energy harvester for broadband concurrent wind and base vibration energy harvesting

\section{Aero-electro-mechanical Model}

The analytical model for the proposed energy harvesting system is established by considering the aero-electro-mechanical coupling behaviors between the structure of the harvester, piezoelectric material and airflow, as well as the impacting behaviors between the harvester and the mechanical stopper. The situation where the harvester and the stopper separately operate without contact is considered first. The analytical model for an aeroelastic piezoelectric energy harvester consists of the electromechanically coupled structural and circuit models as well as the aerodynamic model (Erturk and Inman, 2008; Sirohi and Mahadik, 2012; Zhao et al., 2013; Zhao and Yang, 2015). We consider the harvesting system with the configurations shown in Figure 1, which is excited simultaneously by the base motion $z_{0}(t)$ and the wind flow with a wind speed of $U$. By using the Euler-Bernoulli beam theory, the coupled equation of motion for the harvester is given in Equation (1), while the equation of motion for the stopper is given in Equation (2). The coupled circuit model base on the Gauss law is given in Equation (3).

$$
\begin{gathered}
E I_{h} \frac{\partial^{4} w_{h}\left(x_{h}, t\right)}{\partial x_{h}^{4}}+c_{h}^{s} I_{h} \frac{\partial^{5} w_{h}\left(x_{h}, t\right)}{\partial x_{h}^{4} \partial t}+c_{h}^{a} \frac{\partial w_{h}\left(x_{h}, t\right)}{\partial t}+m_{h} \frac{\partial^{2} w_{h}\left(x_{h}, t\right)}{\partial t^{2}}+\theta V(t)\left[\frac{d \delta\left(x_{h}-x_{1}\right)}{d x_{h}}-\frac{d \delta\left(x_{h}-x_{2}\right)}{d x_{h}}\right] \\
=-\left[m_{h}+M_{t} \delta\left(x_{h}-L_{t}\right)\right] \frac{d^{2} z_{0}(t)}{d t^{2}}+F_{\text {galloping }}(t) \delta\left(x_{h}-L_{t}\right) \\
E I_{s} \frac{\partial^{4} w_{s}\left(x_{s}, t\right)}{\partial x_{s}^{4}}+c_{s}^{s} I_{s} \frac{\partial^{5} w_{s}\left(x_{s}, t\right)}{\partial x_{s}^{4} \partial t}+c_{s}^{a} \frac{\partial w_{s}\left(x_{s}, t\right)}{\partial t}+m_{s} \frac{\partial^{2} w_{s}\left(x_{s}, t\right)}{\partial t^{2}}=-m_{s} \frac{d^{2} z_{0}(t)}{d t^{2}}
\end{gathered}
$$




$$
I(t)+C_{p} \frac{d V(t)}{d t}-\theta \int_{x_{1}}^{x_{2}} \frac{\partial^{3} w_{h}\left(x_{h}, t\right)}{\partial x_{h}^{2} \partial t} d x_{h}=0
$$

Here and hereafter, the subscripts $h$ and $s$ stand for the harvester and stopper beams, respectively. In these equations, $w(x, t)$ is the transverse deflection relative to the base; $I(t)$ and $V(t)$ are, respectively, the current and voltage outputs from the piezoelectric transducer; the terms $E I, c^{s}$, $c^{a}, m, M_{t}$ and $L_{t}$ are, respectively, the flexural rigidity of the beam ( $E$ the elastic modulus and $I$ the equivalent area moment of inertia of the cross section), strain rate damping, viscous air damping, distributed mass of the beam, mass of the bluff body, and position of mass center of the bluff body along the $x_{h}$ axis; $\delta(x)$ is the Dirac delta function; $x_{1}$ and $x_{2}$ denote the start and end positions of the piezoelectric transducer, respectively; $C_{p}$ is the piezoelectric capacitance; and $\theta$ is the electromechanical coupling term which is related to the piezoelectric elastic modulus $E_{p}$, the piezoelectric constant $d_{31}$, the width of the transducer $b_{p}$ and the position $h_{p c}$ of the center of the transducer relative to the neutral axis of the composite cross section by $\theta=-E_{p} d_{31} b_{p} h_{p c}$. The aerodynamic model is built using the quasi-steady hypothesis (Païdoussis et al., 2010). The galloping aerodynamic force $F_{\text {galloping }}(t)$ is given by

$$
F_{\text {galloping }}(t)=\frac{1}{2} \rho h l U^{2} \sum_{i=1}^{3} A_{i} \alpha^{i}, \quad \alpha=\frac{\dot{w}_{h}\left(L_{t}, t\right)+\dot{z}_{0}(t)}{U}+w_{h}^{\prime}\left(L_{t}, \mathrm{t}\right)
$$

where $\rho, h$ and $L$ are, respectively, the air density, diameter and length of the bluff body; $A_{i}$ are empirical aerodynamic coefficients; $\alpha$ is the angle of attack, incorporating the relative wind speed effect and the rotation deformation of the harvester.

The galloping piezoelectric energy harvester has been confirmed to oscillate close to the fundamental frequency in our previous experimental study (Zhao et al., 2013; Zhao and Yang, 2015). In this study, we concern the responses under the base excitation near the fundamental frequency of the harvester. Moreover, the stopper will be oscillating at its own high fundamental frequency due to the impacts during each vibration cycle of the harvester. Therefore, $w_{h}\left(x_{h}, t\right)$ and $w_{s}\left(x_{s}, t\right)$ can be expressed as $w_{h}\left(x_{h}, t\right)=\phi_{h}\left(x_{h}\right) \eta_{h}(t)$ and $w_{s}\left(x_{s}, t\right)=\phi_{s}\left(x_{s}\right) \eta_{s}(t)$, with $\phi(x)$ representing the fundamental mode shape and $\eta(t)$ representing the modal coordinate. Subsequently, the governing equations of the system in Equations (1)-(3) can be rewritten in the modal coordinate as

$$
\begin{gathered}
\ddot{\eta}_{h}(t)+2 \zeta_{h} \omega_{n 1} \dot{\eta}_{h}(t)+\omega_{n 1}{ }^{2} \eta_{h}(t)+\chi V(t)=f_{h, \text { base }}(t)+f_{\text {galloping }}(t) \\
\ddot{\eta}_{s}(t)+2 \zeta_{s} \omega_{n 2} \dot{\eta}_{s}(t)+\omega_{n 2}{ }^{2} \eta_{s}(t)=f_{s, \text { base }}(t) \\
I(t)+C_{p} \dot{V}(t)-\chi \dot{\eta}_{h}(t)=0
\end{gathered}
$$

In these equations, $\zeta$ is the mechanical damping ratio; $\omega_{n 1}$ and $\omega_{n 2}$ are the fundamental frequencies of the harvester and stopper, respectively; $\chi$ is the modal electromechanical coupling coefficient expressed as $\chi=\theta\left[\phi^{\prime}\left(x_{2}\right)-\phi^{\prime}\left(x_{1}\right)\right]$. The modal inertial force $f_{\text {base }}(t)$ due to the base excitation and the modal galloping force $f_{\text {galloping }}(t)$ are obtained as

$$
\begin{gathered}
f_{h, \text { base }}(t)=-\left[\int_{0}^{L_{b}} \phi_{h}\left(x_{h}\right) m_{h} d x_{h}+\phi_{h}\left(L_{t}\right) M_{t}\right] \ddot{z}_{0}(t) \\
f_{s, \text { base }}(t)=-\left[\int_{0}^{L_{s}} \phi_{s}\left(x_{s}\right) m_{s} d x_{s}\right] \ddot{z}_{0}(t) \\
f_{\text {galloping }}(t)=\phi_{h}\left(L_{t}\right) \times \frac{1}{2} \rho h L U^{2}+\sum_{i=1}^{3} A_{i}\left[\frac{\phi_{h}\left(L_{t}\right) \dot{\eta}_{h}(t)+\dot{z}_{0}(t)}{U}+\phi_{h}^{\prime}\left(L_{t}\right) \eta_{h}(t)\right]^{i}
\end{gathered}
$$




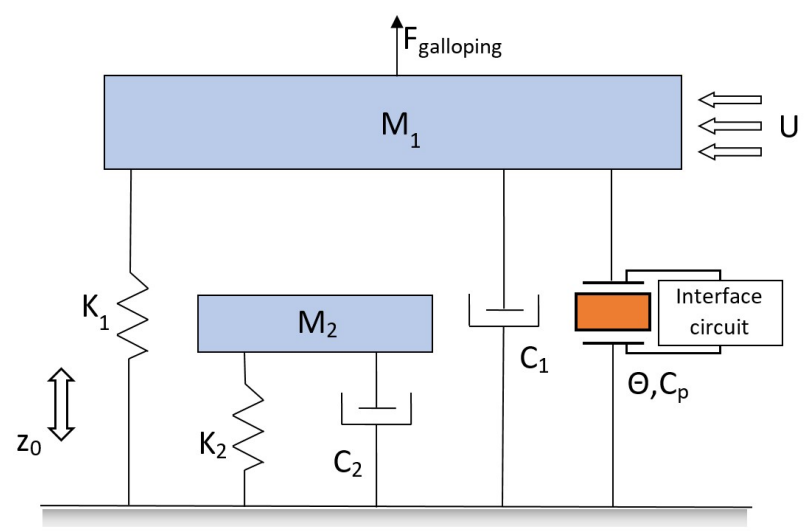

Fig. 2 Schematic of equivalent lumped parameter model

Now, we consider the transformation relations given in Equation (11), which link the parameters in the modal governing equations to the equivalent lumped parameters. Here, $M_{1}, C_{1}$ and $K_{1}$ are the equivalent effective mass, damping and stiffness of the harvester, while $M_{2}, C_{2}$ and $K_{2}$ are the corresponding parameters of the stopper; $\Theta$ is the lumped electromechanical coupling; and $u_{1}(t)$ and $u_{2}(t)$ are the transverse displacements of the bluff body and the stopper at the tip, respectively. Usually, the parameters are selected to make $\omega_{n 2}$ much higher than $\omega_{n 1}$. Therefore, the amplitude of $u_{2}(t)$ is much smaller than that of $u_{1}(t)$ in the concerned frequency range around $\omega_{n 1}$.

$$
\begin{gathered}
M_{1}=\frac{1}{\left[\phi_{h}\left(L_{t}\right)\right]^{2}}, C_{1}=\frac{2 \zeta_{h} \omega_{n 1}}{\left[\phi_{h}\left(L_{t}\right)\right]^{2}}, K_{1}=\frac{\omega_{n 1}{ }^{2}}{\left[\phi_{h}\left(L_{t}\right)\right]^{2}}, \Theta=\frac{\chi}{\phi_{h}\left(L_{t}\right)} \\
M_{2}=\frac{1}{\left[\phi_{s}\left(L_{s}\right)\right]^{2}}, C_{2}=\frac{2 \zeta_{s} \omega_{n 2}}{\left[\phi_{s}\left(L_{s}\right)\right]^{2}}, \quad K_{2}=\frac{\omega_{n 2}{ }^{2}}{\left[\phi_{s}\left(L_{s}\right)\right]^{2}} \\
u_{1}(t)=\eta_{h}(t) \phi_{h}\left(L_{t}\right), u_{2}(t)=\eta_{s}(t) \phi_{s}\left(L_{s}\right)
\end{gathered}
$$

Introducing Equation (11) to the modal governing equations (5)-(7) and the modal forces in (8)(10), the single-mode distributed parameter coupled model can be transformed to the equivalent lumped parameter model given in Equation (12), which is equally effective with the former based on the equivalent parameter substitutions but more convenient for the response analysis. $\beta$ is the ratio of the rotation deformation of the bluff body to the vertical translation expressed as $\beta=\phi^{\prime}\left(L_{t}\right) / \phi\left(L_{t}\right)$; and $\lambda_{1}$ and $\lambda_{2}$ are the correction coefficients of the equivalent lumped inertial forces due to the base excitation for the harvester and stopper, respectively, calculated by Equation (13).

$$
\begin{aligned}
& \left\{\begin{array}{l}
M_{1} \ddot{u}_{1}(t)+C_{1} \dot{u}_{1}(t)+K_{1} u_{1}(t)+\Theta V(t) \\
=-\lambda_{1} M_{1} \ddot{z}_{0}(t)+\frac{1}{2} \rho h L U^{2} \sum_{i=1}^{3}\left[A_{i}\left(\frac{\dot{u}_{1}(t)+\dot{z}_{0}(t)}{U}+\beta u_{1}(t)\right)\right]^{i} \quad\left(u_{1}(t)<D\right)(12) \\
M_{2} \ddot{u}_{2}(t)+C_{2} \dot{u}_{2}(t)+K_{2} u_{2}(t)=-\lambda_{2} M_{2} \ddot{z}_{0}(t) \\
I(t)+C_{p} \dot{V}(t)-\Theta u_{1}(t)=0
\end{array}\right. \\
& \lambda_{1}=\phi_{h}\left(L_{t}\right)\left[\int_{0}^{L_{b}} \phi_{h}\left(x_{h}\right) m_{h} d x_{h}+\phi_{h}\left(L_{t}\right) M_{t}\right], \quad \lambda_{2}=\phi_{s}\left(L_{s}\right)\left[\int_{0}^{L_{s}} \phi_{s}\left(x_{s}\right) m_{s} d x_{s}\right]
\end{aligned}
$$


Figure 2 shows the schematic of the equivalent lumped parameter model. Equation (12) is the aero-electro-mechanically coupled governing equation for the harvesting device when the harvester and stopper vibrate separately without impact. Next, we consider the stage where the bluff body and stopper are engaged. Once the bluff body contacts the stopper, the effective mass, damping and stiffness of the harvester suddenly jump from $M_{1}, C_{1}$ and $K_{1}$ to $M_{1}+M_{2}, C_{1}+C_{2}$ and $K_{1}+K_{2}$, respectively; and at the same time, the difference between $u_{1}(t)$ and $u_{2}(t)$ is constant at $D$ until $M_{1}$ and $M_{2}$ departs. The governing equation is given by

$$
\left\{\begin{array}{l}
\left(M_{1}+M_{2}\right) \ddot{u}_{1}(t)+\left(C_{1}+C_{2}\right) \dot{u}_{1}(t)+\left(K_{1}+K_{2}\right) u_{1}(t)-K_{2} D+\Theta V(t) \\
=-\left(\lambda_{1} M_{1}+\lambda_{2} M_{2}\right) \ddot{z}_{0}(t)+\frac{1}{2} \rho h L U^{2} \sum_{i=1}^{3}\left[A_{i}\left(\frac{\dot{u}_{1}(t)+\dot{z}_{0}(t)}{U}+\beta u_{1}(t)\right)\right]^{i} \quad\left(u_{1}(t) \leq-D\right) \\
u_{2}(t)=u_{1}(t)+D \\
I(t)+C_{p} \dot{V}(t)-\Theta u_{1}(t)=0
\end{array}\right.
$$

In such a way, when the bluff body and the stopper are engaged, the motion of the harvester is controlled by the suddenly increased internal damping $\left(C_{1}+C_{2}\right) \dot{u}_{1}(t)$ and restoring force $\left(K_{1}+K_{2}\right) u_{1}(t)$ along with the backward electromechanical coupling and external aerodynamic and vibratory inertial forces.

\section{Experimental Setup}

Table 1. Properties of cantilever substrate, piezoelectric transducer and mechanical stopper

\begin{tabular}{cccc}
\hline \hline Properties & Cantilever substrate & $\begin{array}{c}\text { Piezoelectric } \\
\text { element }\end{array}$ & $\begin{array}{c}\text { Mechanical } \\
\text { stopper }\end{array}$ \\
\hline Material & Aluminum & MFC M2814-P2 & Aluminum \\
Length $(\mathrm{mm})$ & 135.5 & 28 & 111.5 \\
Width $(\mathrm{mm})$ & 20 & 14 & 40 \\
Thickness $(\mathrm{mm})$ & 0.6 & 0.3 & 2.0 \\
Mass Density $\left(\mathrm{kg} \mathrm{m}^{-3}\right)$ & 2700 & 5440 & 2700 \\
Young's Modulus $(\mathrm{GPa})$ & 69 & 30.336 & 69 \\
Capacitance $(\mathrm{nF})$ & -- & 25.7 & -- \\
Piezoelectric constant $(\mathrm{pm} / \mathrm{V})$ & -- & -170 & -- \\
\hline \hline
\end{tabular}

Table 2. Properties of bluff body

\begin{tabular}{cc}
\hline \hline Properties & Cantilever substrate \\
\hline Material & polystyrene foam \\
Cross section shape & D-shape \\
Length $(\mathrm{mm})$ & 107 \\
Diameter $(\mathrm{mm})$ & 32 \\
Mass $(\mathrm{kg})$ & 0.002 \\
Aerodynamic coefficients & $1.56,0,-6.9$ \\
$A_{1}, A_{2}, A_{3}$ & \\
\hline \hline
\end{tabular}




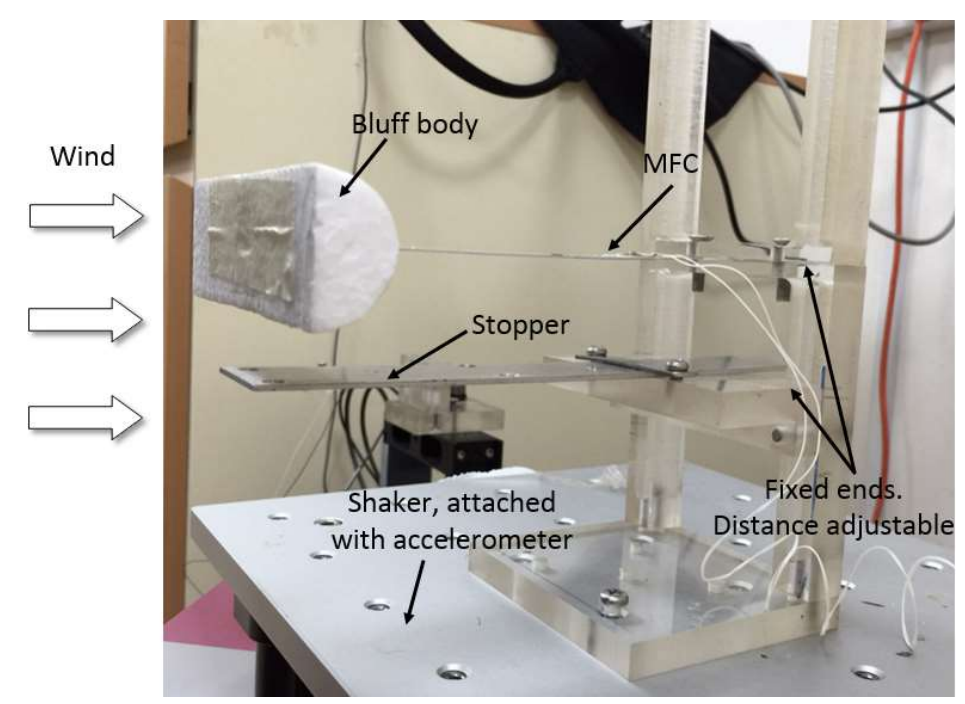

Fig. 3 Experimental setup for proposed energy harvester

Experiment is carried out with a fabricated prototype to evaluate the performance of the proposed design. The experimental setup is shown in Figure 3. The energy harvester consists of an aluminum cantilever beam of which one end is fixed to a rigid support, and a tip body with a Dshaped cross section connected at the free end as a bluff body. To convert the strain energy into electrical charges, a piece of piezoelectric transducer (MFC M2814-P2 from Smart Materials Corp.) is bonded to the top surface of the cantilever near the fixed end, where the largest strain is induced during the oscillation. The piezoelectric capacitance is $25.7 \mathrm{nF}$. The cantilever is of dimension $135.5 \times 20 \times 0.6 \mathrm{~mm}^{3}$. The bluff body is $107 \mathrm{~mm}$ long, with a $\mathrm{D}$-shaped cross section of diameter $32 \mathrm{~mm}$. The weight of the bluff body is $2.0 \mathrm{~g}$. Another aluminum cantilever beam is clamped to the same rigid support to serve as the mechanical stopper, with a width of $40 \mathrm{~mm}$ and a thickness of $2 \mathrm{~mm}$. The free end of the stopper is adjusted to be vertically aligned with the bluff body. The distance between the top surface of the stopper and the bottom surface of the bluff body is $19.5 \mathrm{~mm}$. The properties of the energy harvester prototype are listed in Table 1 and Table 2.

The assembled prototype is then mounted onto an electromagnetic shaker which provides the base vibratory excitation. The vibration acceleration and frequency of the shaker are controlled by a function generator and an amplifier. An accelerometer is attached to the shaker to monitor the acceleration amplitude during the experiment. An axial fan is placed in front of the prototype with the flat surface of the bluff body facing the wind flow. The wind speed is measured with a hotwire anemometer. The air density is taken as $1.204 \mathrm{~kg} / \mathrm{m}^{3}$ as the lab temperature is around $20^{\circ} \mathrm{C}$. The mechanical damping of the harvester is measured using the logarithmic decrement technique by recording the attenuation curves of the short circuit current. To extract the harvested power, certain power extraction interface circuit can be connected across the piezoelectric electrodes for ac-dc conversion and power regulation. In this paper, we focus on the kinetic energy conversion performance of the harvester, therefore, a simple electric circuit is employed which consists of only a resistive load $R$. The voltage $V$ across $R$ is acquired by the NI 9229 DAQ module from National Instruments together with LabVIEW. The current $I$ is calculated by $I=V / R$, and the average power $P_{a v e}$ is calculated by $P_{a v e}=V^{2} / 2 R$. 


\section{Results and Discussion}

\subsection{Experimental validation}

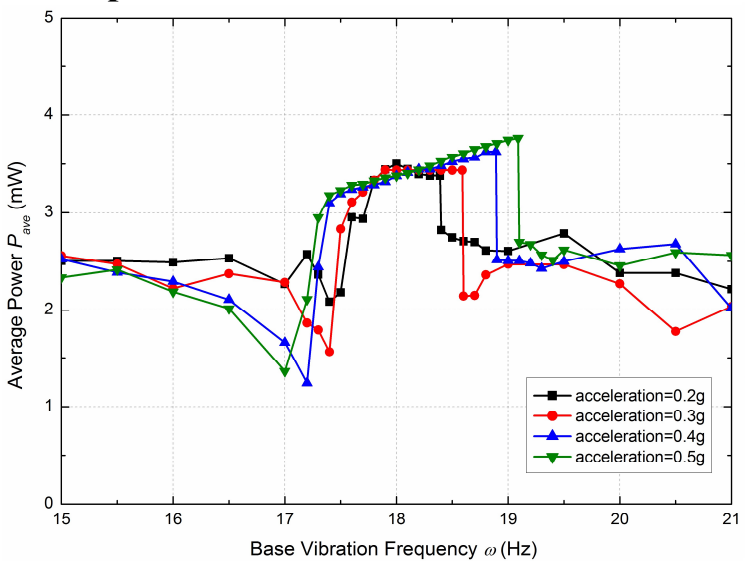

(a)

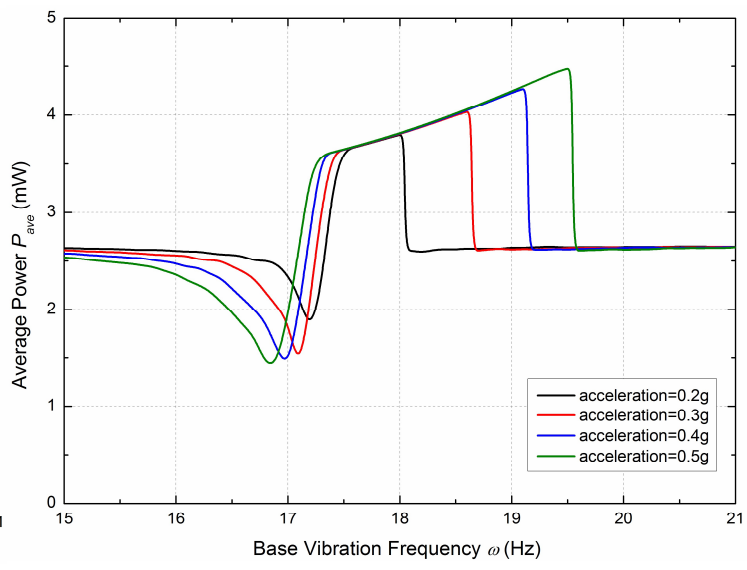

(b)

Fig. 4 Variation of average power with base vibration frequency at a constant wind speed of $5.5 \mathrm{~m} / \mathrm{s}$ and different acceleration levels: (a) experiment, (b) model prediction

Before conducting the test under concurrent wind and base vibration excitations, the empirical aerodynamic coefficients $A_{1} \sim A_{3}$ should be measured first for the wind flow conditions in the present experimental setup. Although many studies in the literature have reported the measured data for these coefficients (Païdoussis et al., 2010), the differences in the flow conditions, e.g., the intensity of turbulence, etc., between our experimental setup and those in the literature will induce extra discrepancy between the measurement and analytical prediction. The prototype is firstly tested under pure aerodynamic excitations by fixing the rigid support into the ground. In such a way, the base vibration acceleration is null. $A_{1} \sim A_{3}$ are calculated by matching the predicted cutin speed and voltage amplitudes in the considered wind speed range with the experimental measurements. First, the cut-in wind speed $U_{c r}$ is measured at a short circuit condition. Depending on the relation between $U_{c r}$ and $A_{1}$ as derived by Zhao and Yang (2015), $A_{1}$ is calculated by

$$
A_{1}=\frac{4 \omega_{n} M \zeta}{U_{c r} \rho h L}
$$

$A_{2}$ is zero according to the characteristics of translational galloping (Païdoussis et al., 2010; Barrero-Gil et al., 2010). Next, the voltage responses are measured at a range of wind speeds with a connected resistor of $275 \mathrm{k} \Omega$. Numerical simulations are conducted based on Equation (12) with the already determined parameters while $\ddot{z}_{0}(t)$ is set to be $0 . A_{3}$ is then identified by matching the predicted voltage amplitudes with the experimental measurements. It is determined that $A_{1}=1.56, A_{2}=0$, and $A_{3}=-6.9$.

Figure 4 shows the variations of the average power as a function of the base vibration frequency at different acceleration levels from the experiment and analytical prediction. The wind speed is constant at $5.5 \mathrm{~m} / \mathrm{s}$. It is seen from both experiment and analysis that, for all considered acceleration values, when the base vibration frequency is far away from the resonance of $17.6 \mathrm{~Hz}$, the average output power curve is generally flat, with almost the same power amplitude as that from pure galloping at $5.5 \mathrm{~m} / \mathrm{s}$, that is, around $2.4 \mathrm{~mW}$. This result is consistent with the previous 
studies on concurrent wind and vibration energy harvesting with linear aeroelastic energy harvesters (Bibo and Daqaq, 2013b; Bibo et al., 2015). In this situation, the base vibration energy is barely harnessed by the energy harvester. As the base vibration frequency sweeps up to be slightly lower than the resonance, the output power gradually decreases to a minimum. Subsequently, as the base vibration frequency increases toward the resonance, the power sharply increases until the bluff body impacts the mechanical stopper. This corresponds to the case when the difference between the displacement of the bluff body and that of the stopper at the tip achieves the original stopper-bluff body distance $\mathrm{D}$, which is $19.5 \mathrm{~mm}$ in the present case. The output power continuously increases over a wide range of base vibration frequencies, within which both wind energy and base vibration energy are effectively harnessed by the harvester. The power increasing ratio within this range is small due to the high effective stiffness when the harvester impacts the stopper, i.e., $K_{1}+K_{2}$ in Equation (2). In fact, according to our observation during the experiment, the stopper works more like a rigid enclosure base with negligible deflections. The influence of the stiffness as well as other parameters of the stopper on the power response will be investigated later in Section 5.3. The bluff body stops engaging the stopper when the base vibration frequency increases to higher values, and the output power drops back to the level of pure galloping energy harvesting.

In this paper, we define the bandwidth of concurrent wind and base vibration energy harvesting as the frequency range within which both wind energy and base vibration energy are effectively harnessed. The experiment clearly demonstrates the broad bandwidth of concurrent energy harvesting by employing a galloping aeroelastic energy harvester integrated with the mechanical stopper. The widest bandwidth is achieved at a base acceleration of $0.5 \mathrm{~g}$, with the output power steadily increases from $3.0 \mathrm{~mW}$ at $17.3 \mathrm{~Hz}$ to $3.8 \mathrm{~mW}$ at $19.1 \mathrm{~Hz}$. Overall, reasonable agreement is obtained between the experimental and analytical results. The analysis captures the trends of the responses very well, yet the power amplitudes are over predicted. The discrepancies may be mainly due to the inevitable energy losses during the impacts, which are not considered in the simulation. Especially, it can be deduced that the energy loss gets higher at higher frequency, therefore, the discrepancy is more obvious at the right edges of the bandwidths.

\subsection{Performance comparison}

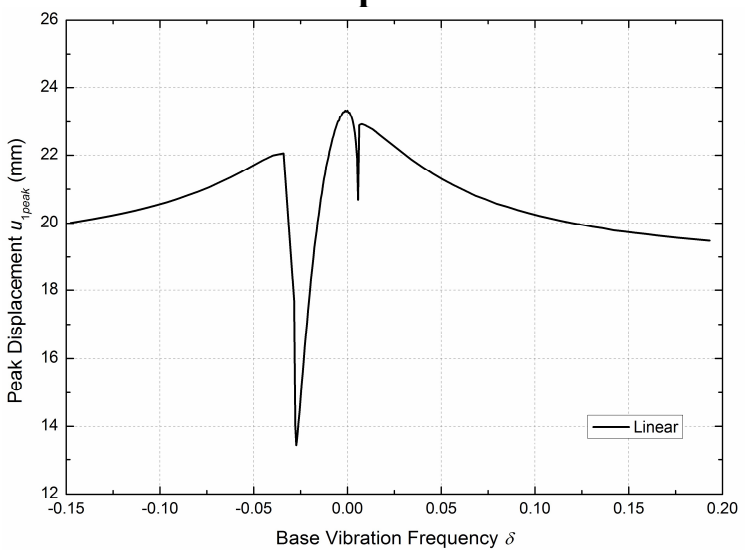

(a)

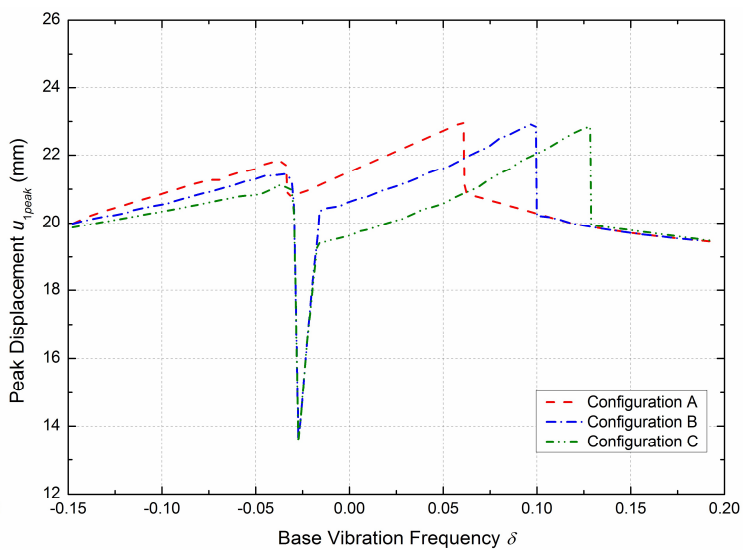

(b) 
This article has been published as "Zhao, L., \& Yang, Y. (2018). An impact-based broadband aeroelastic energy harvester for concurrent wind and base vibration energy harvesting. Applied Energy, 212, 233243" with doi: 10.1016/j.apenergy.2017.12.042

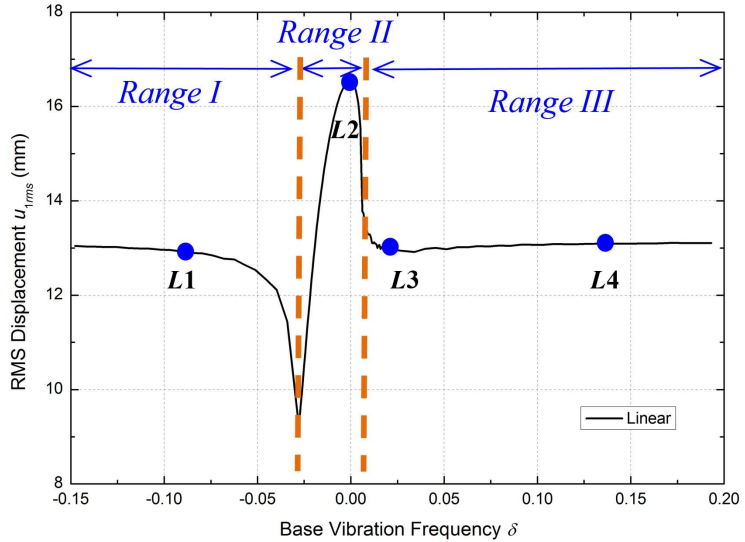

(c)

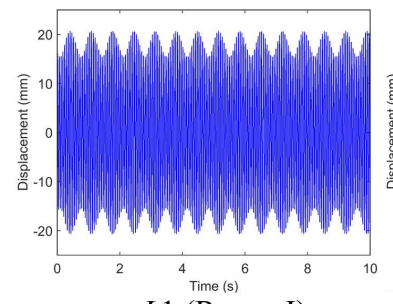

L1 (Range I)

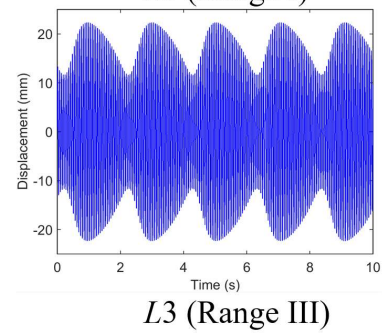

(e)

e)

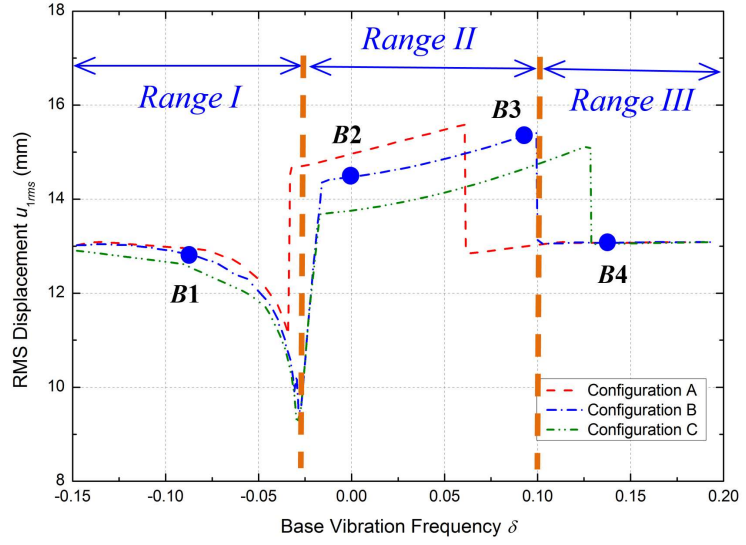

(d)

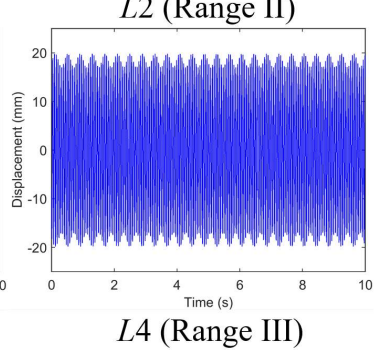

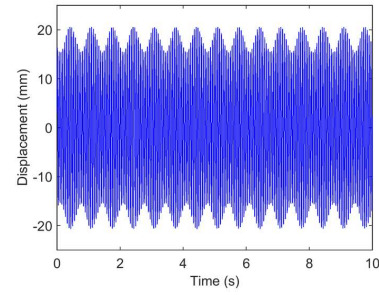

$B 1$ (Range I)

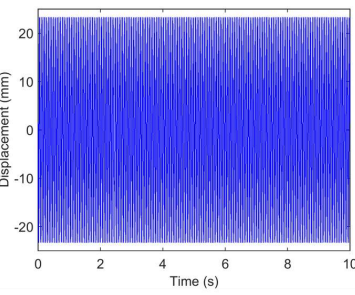

L2 (Range II)

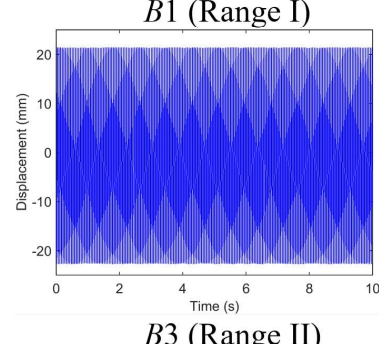

B3 (Range II)

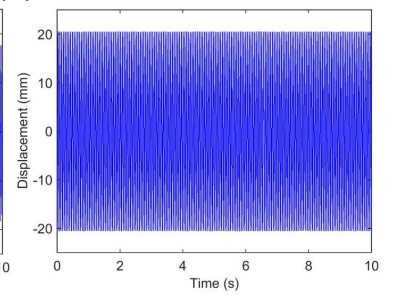

$B 2$ (Range II)

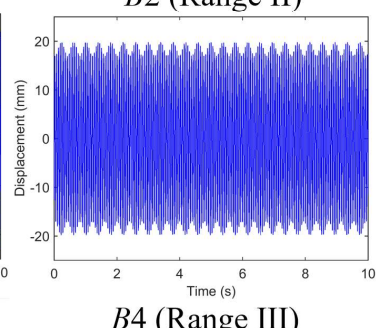

(f)

Fig. 5 Response of displacement at a wind speed of $6 \mathrm{~m} / \mathrm{s}$ and a base acceleration of $0.3 \mathrm{~g}$ : (a) (b) Frequency response of peak displacement of bluff body; (c)(d) frequency response of RMS displacement of bluff body; (e)(f) time domain response. (a)(c)(e): conventional harvester; (b)(d)(f) proposed harvester.

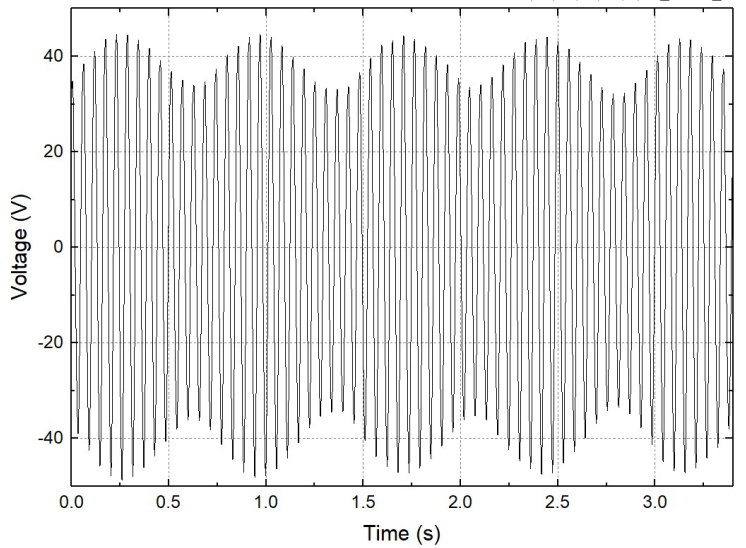

(a)

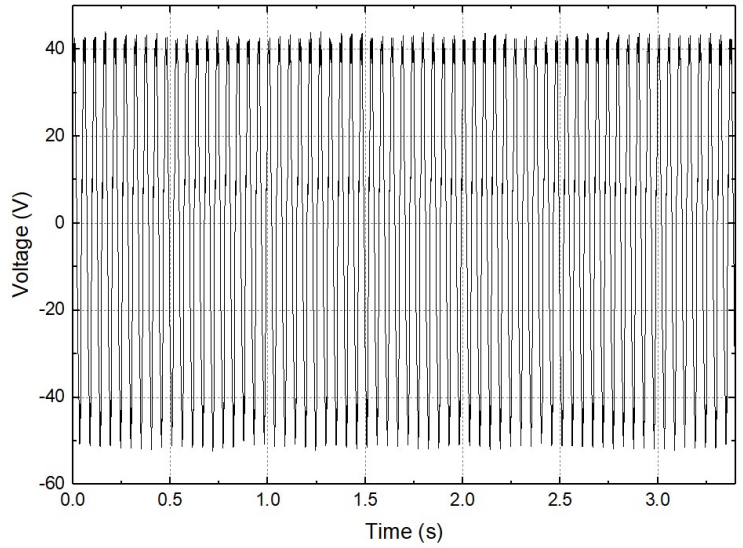

(b) 
Fig.6 Experimental time domain response of generated voltage at a wind speed of $5.5 \mathrm{~m} / \mathrm{s}$, a base acceleration of $0.5 \mathrm{~g}$, and a base vibration frequency of $19 \mathrm{~Hz}$ : (a) conventional harvester; (b) proposed harvester.

In order to further understand the behavior of the proposed design of a broadband concurrent energy harvester with a mechanical stopper, and to compare it with that of a conventional linear concurrent energy harvester, we plot the displacement responses of the bluff body in Figure 5 based on the model analysis, including frequency responses of the peak displacement and root mean square (RMS) displacement as well as the time domain response. The variation of the peak displacement as a function of the non-dimensional base vibration frequency $\delta$ from the conventional harvester is plotted in Figure 5(a), while that from the proposed harvester is shown in Figure 5(b). The corresponding variations of the RMS displacement are shown in Figure 5(c) and (d), respectively. $\delta$ is calculated by $\left(\omega-\omega_{n 1}\right) / \omega_{n 1}$ where $\omega$ is the base vibration frequency and $\omega_{n 1}$ is the fundamental frequency of the aeroelastic energy harvester. Three stopper configurations are considered. For configuration $\mathrm{A}$, it is chosen that $D=20 \mathrm{~mm}$ and $M_{2}=8.0 \mathrm{~g}$ with $\omega_{n 2}=96.8 \mathrm{~Hz}$; for configuration $\mathrm{B}$, it is chosen that $D=20 \mathrm{~mm}$ and $M_{2}=2.9 \mathrm{~g}$ with $\omega_{n 2}=160 \mathrm{~Hz}$; and for configuration $\mathrm{C}$, it is chosen that $\mathrm{D}=19 \mathrm{~mm}$ and $M_{2}=2.9 \mathrm{~g}$ with $\omega_{n 2}=160 \mathrm{~Hz}$. The base acceleration is constant at $0.3 \mathrm{~g}$, and the wind speed is constant at $6 \mathrm{~m} / \mathrm{s}$. Inspecting Figure 5(a) and (c), it is seen that the response of a conventional concurrent energy harvester can be divided into three regions, as indicated in Figure 5(c). In Region I, the peak displacement first gradually increases with $\delta$ to $22.0 \mathrm{~mm}$, then it sharply drops to a minimum value of $13.4 \mathrm{~mm}$. In contrast, the RMS displacement in Region I monotonically decreases with increasing $\delta$. In Region II, both the peak and RMS displacements are very sensitive to the frequency deviation. The peak and RMS displacements quickly increase with $\delta$ and reach the respective maximum values of $23.3 \mathrm{~mm}$ and $16.5 \mathrm{~mm}$ at $\delta=0$, i.e., the resonance. After that, they quickly drop when $\delta$ goes beyond 0 . In Region III, the RMS displacement slightly decreases at the left edge of the region and then keeps constant at $13.1 \mathrm{~mm}$ across the most range of $\delta$. On the contrary, the peak displacement sharply returns to a high value of $22.9 \mathrm{~mm}$ at the left edge of Region III, and then slowly decreases with the increase of $\delta$.

To better understand the behaviors in time domain, we choose four representative points for the conventional linear concurrent energy harvester, as indicated in Figure 5(c), and plot the steady state time domain responses at the corresponding frequencies, as shown in Figure 5(e). It is seen that at point $L 1$ within Region I where $\delta=-0.0909$, due to the two different frequencies from the aerodynamic excitation and the base excitation, the response is quasi-periodic with an amplitude envelope at a modulation frequency. At point $L 2$ within Region II where $\delta=0$, the two frequencies lock into each other and the response is periodic. This corresponds to the situation where the two types of energy sources are effectively harnessed. However, when $\delta$ is slightly over 0 , e.g., $\delta=0.0227$ at point $L 3$ in Region III, the response is quasi-periodic again, with large amplitude modulation. At this point, although there is a high peak displacement amplitude of $22.4 \mathrm{~mm}$, the RMS value is low at $12.9 \mathrm{~mm}$. This is disadvantageous for the purpose of energy harvesting since the beam experiences large deflection yet fails to give the expected effective power output. Finally, at point $L 4$ within Region III where $\delta=0.1364$, quasi-periodic oscillation is obtained with minor amplitude modulation. 
In contrast, the proposed broadband design shows different behaviors. Similarly, we divide the response of the proposed design into three regions, as indicated in Figure 5(d) for configuration $\mathrm{B}$ as an example. In Region I, the variation of the peak and RMS displacements shows similar trends with that of the conventional harvester. However, in Region II, different from the case of the conventional harvester with great sensitivity to the frequency deviation, the peak and RMS displacements of the proposed design stay in relatively high values over a wide range of $\delta$ before they drop and enter Region III. For example, for configuration B, the RMS displacement increases steadily from $14.4 \mathrm{~mm}$ to $15.4 \mathrm{~mm}$ when $\delta$ increases from -0.0159 to 0.0994 ; while the corresponding peak displacement increases steadily from $20.4 \mathrm{~mm}$ to $23.0 \mathrm{~mm}$. The time domain responses at steady state for the four representative points $B 1-B 4$ are plotted in Figure 5(f). The responses of point $B 1$ of Region I ( $\delta=-0.0909)$ and $B 4$ of Region III $(\delta=0.1364)$ are quasi-periodic and identical to the case of the conventional harvester. At point $B 2$ with $\delta=0$, the harvester has engaged the mechanical stopper and the response is periodic. It is worth noting that at point B3 where $\delta$ has been increased to 0.0909 , periodic response still persists due to the introduction of the mechanical stopper, forcing the two excitation frequencies to lock into each other. As a result, broadened bandwidth for effective concurrent energy harvesting is achieved, and at the same time, the peak beam deflections are slightly mitigated and fully utilized for power conversion. All three stopper configurations achieve broadband concurrent energy harvesting. Compared to configuration B, configuration A obtains higher displacement amplitudes and narrower bandwidth, while configuration $\mathrm{C}$ achieves broader bandwidth and lower amplitudes. Figure 6 further plots the time domain response of voltage across the piezoelectric electrodes measured from the experiment, at a constant wind speed of $5.5 \mathrm{~m} / \mathrm{s}$, a base acceleration of $0.5 \mathrm{~g}$, and a base vibration frequency of $19 \mathrm{~Hz}$. The connected load resistance is $275 \mathrm{k} \Omega$. It is clearly shown that with the mechanical stopper, the original quasi-periodic oscillations of a conventional harvester are converted to periodic vibrations.

\subsection{Influence of stopper configurations and excitation strengths}

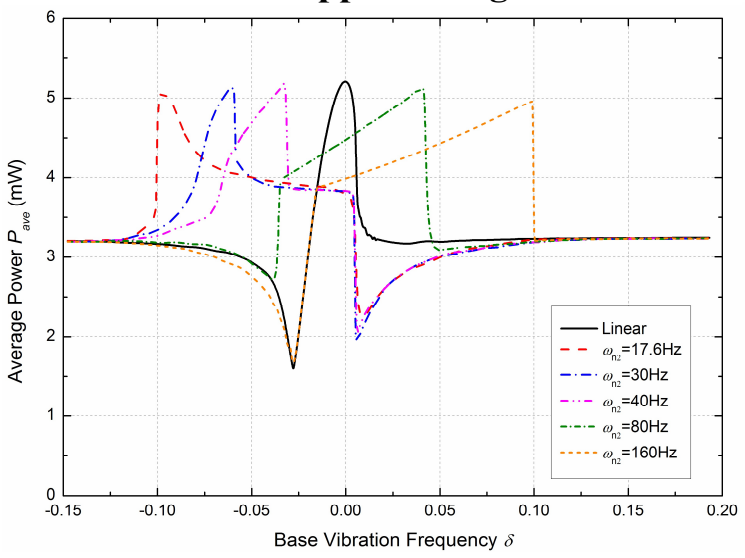

(a)

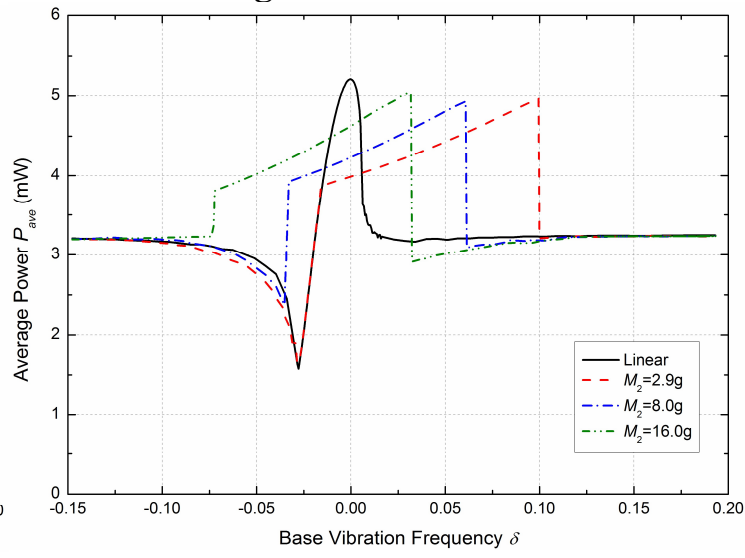

(b) 


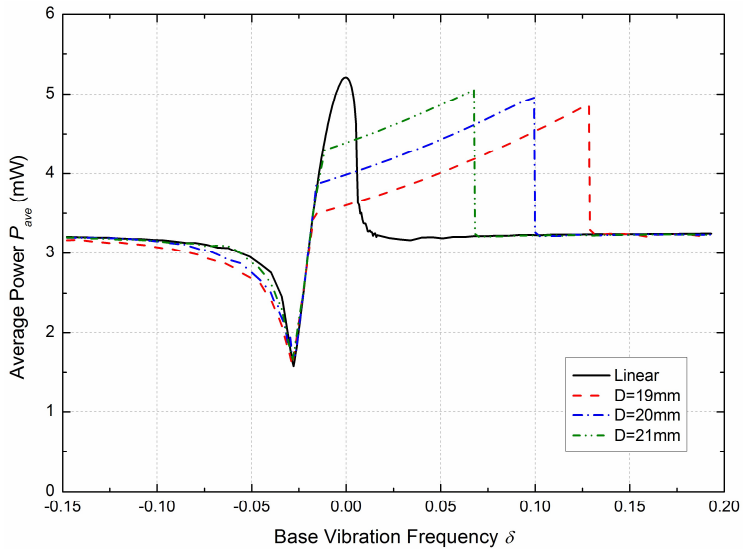

(c)

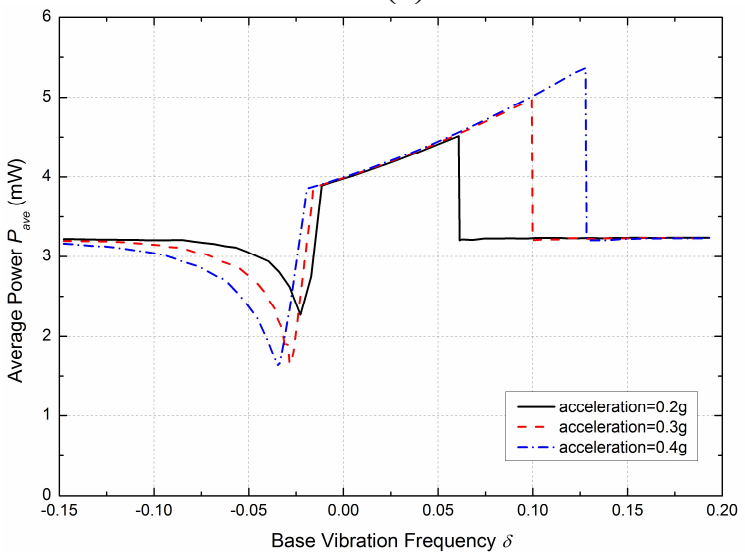

(e)

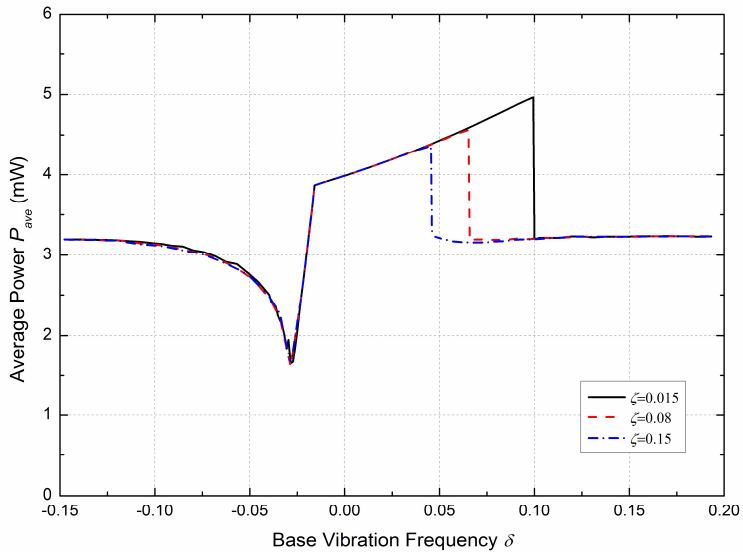

(d)

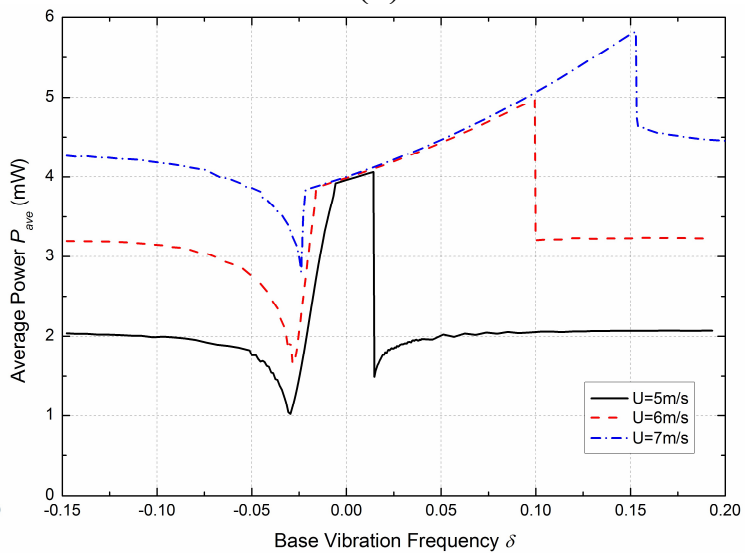

(f)

Fig. 7 Variation of average power $P_{\text {ave }}$ with base vibration frequency $\delta$ at different (a) fundamental frequencies of stopper, (b) effective masses of stopper, (c) stopper-bluff body distances, (d) mechanical damping values of stopper, (e) base vibration accelerations and (f) wind speeds.

In view of the different performances of the three stopper configurations in Section 5.2, we carry out a parametric study to investigate the influences of several system parameters on the efficiency of concurrent energy harvesting. These parameters include the fundamental frequency of the stopper (stiffness), effective mass of the stopper, distance between the stopper free end and the bluff body, and the mechanical damping of the stopper. The effects of the excitation strengths including the base vibration acceleration and wind speed on the energy harvesting performance are also studied.

The variation of average power $P_{\text {ave }}$ with base vibration frequency $\delta$ for different fundamental frequencies of the stopper $\omega_{n 2}$ is shown in Figure 7(a). $\omega_{n 2}$ is varied by changing the stiffness of the stopper $K_{2}$ while keeping the other parameters constant. It is noted that with the increase of $\omega_{n 2}\left(K_{2}\right)$, the bandwidth for effectively concurrent energy harvesting becomes wider and shifts to the right. Moreover, the trend of the response curve varies with $\omega_{n 2}$. With smaller $\omega_{n 2}$ of $17.6 \mathrm{~Hz}$, $30 \mathrm{~Hz}$ and $40 \mathrm{~Hz}$, there is a valley in the power response at the right side of the bandwidth range, while with higher $\omega_{n 2}$ of $80 \mathrm{~Hz}$ and $160 \mathrm{~Hz}$, the power valley appears at the left side. Inspecting 
the response within the bandwidth, it is found that the power increasing ratio decreases with increasing $\omega_{n 2}$. However, no much difference in the peak power magnitude is observed. Next, we keep the stiffness of the stopper as well as other parameters of the stopper fixed, and vary the effective mass $M_{2}$. This can be achieved by adding a proof mass to the stopper. The power variation with varying $M_{2}$ is depicted in Figure 7(b). It is seen that the effective concurrent energy harvesting bandwidth shifts to the left with the increase of $M_{2}$. The influence of $M_{2}$ on the bandwidth and the peak power magnitude is not obvious. Nevertheless, it greatly affects the power valley behaviour at the left side of the bandwidth. The larger the $M_{2}$ is, the shallower the valley is, which is of great benefits for the purpose of energy harvesting. It can be explained that with a larger proof mass, the mechanical stopper has larger vertical displacement as compared to the case with no proof mass or with a smaller proof mass. As a result, it can engage the bluff body before its movement enters the valley, i.e., before the peak displacement of the bluff body drops at $\delta=-$ 0.0341 as shown in Figure 5(a), thus removing the subsequent valley in the response. However, the associated drawback is that the weight power density, i.e., power per unit weight, of the overall harvesting device will be reduced by adding a large proof mass to the stopper.

The influence of the stopper-bluff body distance $D$ on the power response is depicted in Figure 7(c). With the increase of $D$, the bandwidth gets narrower, while the power amplitude gets higher. As for the effects of the damping of the stopper $\zeta$, it is seen from Figure 7(d) that both the bandwidth and the peak power magnitude decrease with increasing $\zeta$. Figure 7(e) shows the power variation at different base vibration accelerations. As can be seen, the acceleration does not affect the response when the base vibration frequency is far away from the resonance. In order to achieve larger bandwidth, higher acceleration is demanded, because both the bandwidth and the peak power magnitude increase with acceleration. Finally, the influence of the wind speed on the power response is revealed in Figure 7(f). It is noted that at the regions away from the engaging state, the power increases with wind speed, which is reasonable since the power level from these regions corresponds to that from the pure galloping condition. Within the bandwidth where the bluff body impacts the stopper, the output power at different wind speeds increases with the same power increasing ratio. With the increase of wind speed, the bandwidth for effective concurrent energy harvesting gets wider, and the achievable maximum power level gets higher. However, the power enhancement within the bandwidth as compared to the non-impact regions decreases with increasing wind speed at such a condition with a constant stopper-bluff body distance.

In summary, for the purpose of concurrent base vibration and wind energy harvesting, the increase of the stiffness of the mechanical stopper, base vibration acceleration and wind speed is beneficial, enabling broadened bandwidth and increased peak power. Moreover, increasing the effective mass of the stopper by adding a proof mass can benefit the overall concurrent energy harvesting performance by removing the power valley, yet at the same time reduce the power density in weight. A smaller stopper-bluff body distance produces a wider bandwidth yet slightly reduces the peak power, therefore, there is a trade-off for the optimum distance value. Finally, although it is hard to control the damping of the stopper in a practical situation, it should be reduced as much as possible for the benefit of concurrent energy harvesting.

\section{Conclusion}

In this paper, a novel energy harvester which concurrently harnesses energy from base vibrations and wind flows is proposed by utilizing a mechanical stopper to achieve a broadened bandwidth. 
The quasi-periodic oscillations are converted to periodic vibration due to the introduction of the mechanical stopper which forces the two excitation frequencies from wind and base vibration to lock into each other. An aero-electro-mechanically coupled model is established by considering the mutual coupling behaviors between the harvester structure, piezoelectric transducer and airflow, and the impacting behaviors between the harvester and the mechanical stopper. Experiment is carried out for validation with a fabricated prototype. Broadened bandwidth for effective concurrent energy harvesting is achieved while the peak beam deflections are slightly mitigated and fully utilized for power conversion. At a wind speed of $5.5 \mathrm{~m} / \mathrm{s}$ and a base acceleration of $0.5 \mathrm{~g}$, the output power from the proposed harvesting device steadily increases from $3.0 \mathrm{~mW}$ at $17.3 \mathrm{~Hz}$ to $3.8 \mathrm{~mW}$ at $19.1 \mathrm{~Hz}$.

Guidelines for the stopper configuration are proposed based on a parametric study for enhanced performance of broadband wind and vibration energy harvesting. It is recommended that the stiffness of the stopper, wind speed and acceleration of base excitation should be set as high as possible, while the damping of the stopper should be kept low. A relatively larger effective mass of the stopper can remove the power valley yet with a sacrifice of power density in weight. There is a trade-off for the optimum stopper-bluff body distance since reducing the distance broadens the bandwidth yet simultaneously decreases the peak power. Generally, with the increase of the wind speed, the distance should be accordingly increased. It is concluded that the proposed design of integrating an aeroelastic energy harvester with a mechanical stopper is a viable solution for broadband concurrent wind and base vibration energy harvesting.

\section{REFERENCES}

Abdelkefi, A., Scanlon J.M., McDowell E. and Hajj M.R. (2013), "Performance enhancement of piezoelectric energy harvesters from wake galloping". Applied Physics Letters, 103, 033903.

Ahmed, A., Hassan, I., Hedaya, M., El-Yazid, T. A., Zu, J. and Wang, Z. L. (2017), "Farms of triboelectric nanogenerators for harvesting wind energy: A potential approach towards green energy". Nano Energy, 36, 21-29.

Akaydin, H.D., Elvin N. and Andreopoulos Y. (2012), "The performance of a self-excited fluidic energy harvester". Smart Materials and Structures, 21, 025007.

Aquino, A.I., Calautit J.K. and Hughes B.R. (2017), "Evaluation of the integration of the Wind-Induced Flutter Energy Harvester (WIFEH) into the built environment: Experimental and numerical analysis". Applied Energy.

Barrero-Gil, A., Alonso G. and Sanz-Andres A. (2010), "Energy harvesting from transverse galloping". Journal of Sound and Vibration, 329, 2873-2883.

Bibo, A., Abdelkefi A. and Daqaq M.F. (2015), "Modeling and characterization of a piezoelectric energy harvester under Combined Aerodynamic and Base Excitations". Journal of Vibration and Acoustics, 137, 031017.

Bibo, A. and Daqaq M.F. (2013a), "Energy harvesting under combined aerodynamic and base excitations". Journal of Sound and Vibration, 332, 5086-5102.

Bibo, A. and Daqaq M.F. (2013b), "Investigation of concurrent energy harvesting from ambient vibrations and wind using a single piezoelectric generator". Applied Physics Letters, 102, 243904.

Bryant, M. and Garcia E. (2011), "Modeling and Testing of a Novel Aeroelastic Flutter Energy Harvester". Journal of Vibration and Acoustics, 133, 011010.

Dai, H., Abdelkefi, A., Javed, U. and Wang, L. (2015), "Modeling and performance of electromagnetic energy harvesting from galloping oscillations". Smart Materials and Structures, 24, 045012.

Dai, H.L., Abdelkefi A. and Wang L. (2014), "Piezoelectric energy harvesting from concurrent vortexinduced vibrations and base excitations". Nonlinear Dynamics, 77, 967-981. 
This article has been published as "Zhao, L., \& Yang, Y. (2018). An impact-based broadband aeroelastic energy harvester for concurrent wind and base vibration energy harvesting. Applied Energy, 212, 233243" with doi: 10.1016/j.apenergy.2017.12.042

Dai, H. L., Abdelkefi, A., Yang, Y. and Wang, L. (2016), "Orientation of bluff body for designing efficient energy harvesters from vortex-induced vibrations". Applied Physics Letters, 108, 053902.

Daqaq, M.F., Masana R., Erturk A. and Quinn D.D. (2014), "On the role of nonlinearities in vibratory energy harvesting: a critical review and discussion". Applied Mechanics Reviews, 66, 040801.

Den Hartog, J.P. (1956) Mechanical Vibrations, New York: McGraw-Hill.

Erturk, A. and Inman D.J. (2008), "On mechanical modeling of cantilevered piezoelectric vibration energy harvesters". Journal of Intelligent Material Systems and Structures, 19, 1311-1325.

Ewere, F., Wang G. and Cain B. (2014), "Experimental investigation of galloping piezoelectric energy harvesters with square bluff bodies". Smart Materials and Structures, 23, 104012.

$\mathrm{Fu}, \mathrm{H}$. and Yeatman E.M. (2017), "A methodology for low-speed broadband rotational energy harvesting using piezoelectric transduction and frequency up-conversion". Energy, 125, 152-161.

Goushcha, O., Elvin N. and Andreopoulos Y. (2014), "Interactions of vortices with a flexible beam with applications in fluidic energy harvesting". Applied Physics Letters, 104, 021919.

$\mathrm{Gu}$, L. and Livermore C. (2011), "Impact-driven, frequency up-converting coupled vibration energy harvesting device for low frequency operation". Smart Materials and Structures, 20, 045004.

Harne, R. and Wang K. (2013), "A review of the recent research on vibration energy harvesting via bistable systems". Smart Materials and Structures, 22, 023001.

Hobeck, J.D. and Inman D.J. (2014), "A distributed parameter electromechanical and statistical model for energy harvesting from turbulence-induced vibration". Smart Materials and Structures, 23, 115003.

Jung, I., Shin Y.-H., Kim S., Choi J.-y. and Kang C.-Y. (2017), "Flexible piezoelectric polymer-based energy harvesting system for roadway applications". Applied Energy, 197, 222-229.

Kim, S., Ju S., Ji C. and Lee S. (2014) Equivalent circuit model of an impact-based piezoelectric energy harvester. Journal of Physics: Conference Series. IOP Publishing, 012094.

Li, S., Wang, J., Peng, W., Lin, L., Zi, Y., Wang, S., Zhang, G. and Wang, Z. L. (2017), "Sustainable Energy Source for Wearable Electronics Based on Multilayer Elastomeric Triboelectric Nanogenerators". Advanced Energy Materials, 7, 1602832.

Liu, H., Lee C., Kobayashi T., Tay C.J. and Quan C. (2012), "Investigation of a MEMS piezoelectric energy harvester system with a frequency-widened-bandwidth mechanism introduced by mechanical stoppers". Smart Materials and Structures, 21, 035005.

Liu, H., Tay C.J., Quan C., Kobayashi T. and Lee C. (2011), "A scrape-through piezoelectric MEMS energy harvester with frequency broadband and up-conversion behaviors". Microsystem technologies, 17, 1747-1754.

McCarthy, J., Watkins S., Deivasigamani A. and John S. (2016), "Fluttering energy harvesters in the wind: A review". Journal of Sound and Vibration, 361, 355-377.

Morbiato, T., Borri C. and Vitaliani R. (2014), "Wind energy harvesting from transport systems: A resource estimation assessment". Applied Energy, 133, 152-168.

Naseer, R., Dai H., Abdelkefi A. and Wang L. (2017), "Piezomagnetoelastic energy harvesting from vortex-induced vibrations using monostable characteristics". Applied Energy, 203, 142-153.

Orrego, S., Shoele K., Ruas A., Doran K., Caggiano B., Mittal R. and Kang S.H. (2017), "Harvesting ambient wind energy with an inverted piezoelectric flag". Applied Energy, 194, 212-222.

Païdoussis, M.P., Price S.J. and De Langre E. (2010) Fluid-structure interactions: Cross-flow-induced instabilities, New York: Cambridge University Press.

Phan, H., Shin, D.-M., Jeon, S. H., Kang, T. Y., Han, P., Kim, G. H., Kim, H.K., Kim, K., Hwang, Y.H. and Hong, S.W. (2017), "Aerodynamic and aeroelastic flutters driven triboelectric nanogenerators for harvesting broadband airflow energy". Nano Energy, 33, 476-484.

Sirohi, J. and Mahadik R. (2012), "Harvesting Wind Energy Using a Galloping Piezoelectric Beam". Journal of Vibration and Acoustics, 134, 011009.

Soliman, M., Abdel-Rahman E., El-Saadany E. and Mansour R. (2008), "A wideband vibration-based energy harvester". Journal of Micromechanics and Microengineering, 18, 115021. 
This article has been published as "Zhao, L., \& Yang, Y. (2018). An impact-based broadband aeroelastic energy harvester for concurrent wind and base vibration energy harvesting. Applied Energy, 212, 233243" with doi: 10.1016/j.apenergy.2017.12.042

Tang, L. and Yang Y. (2012), "A multiple-degree-of-freedom piezoelectric energy harvesting model". Journal of Intelligent Material Systems and Structures, 23, 1631-1647.

Tang, L. and Yang Y. (2012), "A nonlinear piezoelectric energy harvester with magnetic oscillator". Applied Physics Letters, 101, 094102.

Vicente-Ludlam, D., Barrero-Gil A. and Velazquez A. (2015), "Enhanced mechanical energy extraction from transverse galloping using a dual mass system". Journal of Sound and Vibration, 339, 290 303.

Vocca, H., Neri I., Travasso F. and Gammaitoni L. (2012), "Kinetic energy harvesting with bistable oscillators". Applied Energy, 97, 771-776.

Wang, X., Chen C., Wang N., San H., Yu Y., Halvorsen E. and Chen X. (2017), "A frequency and bandwidth tunable piezoelectric vibration energy harvester using multiple nonlinear techniques". Applied Energy, 190, 368-375.

Wu, Y., Badel A., Formosa F., Liu W. and Agbossou A. (2014), "Nonlinear vibration energy harvesting device integrating mechanical stoppers used as synchronous mechanical switches". Journal of Intelligent Material Systems and Structures, 25, 1658-1663.

Wu, Y., Li D., Xiang J. and Da Ronch A. (2017), "Piezoaeroelastic energy harvesting based on an airfoil with double plunge degrees of freedom: Modeling and numerical analysis". Journal of Fluids and Structures, 74, 111-129.

Yan, Z., Abdelkefi A. and Hajj M.R. (2014), "Piezoelectric energy harvesting from hybrid vibrations". Smart Materials and Structures, 23, 025026.

Yang, W. and Towfighian S. (2017), "A hybrid nonlinear vibration energy harvester". Mechanical Systems and Signal Processing, 90, 317-333.

Yang, Z. and Zu J. (2016), "Toward harvesting vibration energy from multiple directions by a nonlinear compressive-mode piezoelectric transducer". IEEE/ASME Transactions on Mechatronics, 21, 1787-1791.

Young, J., Lai J.C. and Platzer M.F. (2014), "A review of progress and challenges in flapping foil power generation". Progress in Aerospace Sciences, 67, 2-28.

Zhang, L., Abdelkefi, A., Dai, H., Naseer, R. and Wang, L. (2017a), "Design and experimental analysis of broadband energy harvesting from vortex-induced vibrations". Journal of Sound and Vibration, 408, 210219.

Zhang, L., Dai, H., Abdelkefi, A. and Wang, L. (2017b), "Improving the performance of aeroelastic energy harvesters by an interference cylinder". Applied Physics Letters, 111, 073904.

Zhang, X., Pan H., Qi L., Zhang Z., Yuan Y. and Liu Y. (2017c), "A renewable energy harvesting system using a mechanical vibration rectifier (MVR) for railroads". Applied Energy.

Zhang, Z., Zhang X., Rasim Y., Wang C., Du B. and Yuan Y. (2016), "Design, modelling and practical tests on a high-voltage kinetic energy harvesting $(\mathrm{EH})$ system for a renewable road tunnel based on linear alternators". Applied Energy, 164, 152-161.

Zhao, L., Tang L., Liang J. and Yang Y. (2017), "Synergy of wind energy harvesting and synchronized switch harvesting interface circuit". IEEE/ASME Transactions on Mechatronics, 22, 1093-1103.

Zhao, L., Tang L. and Yang Y. (2013), "Comparison of modeling methods and parametric study for a piezoelectric wind energy harvester". Smart Materials and Structures, 22, 125003.

Zhao, L., Tang L. and Yang Y. (2014), "Enhanced piezoelectric galloping energy harvesting using 2 degree-of-freedom cut-out cantilever with magnetic interaction". Japanese Journal of Applied Physics, 53, 060302.

Zhao, L., Tang L. and Yang Y. (2016), "Synchronized charge extraction in galloping piezoelectric energy harvesting". Journal of Intelligent Material Systems and Structures, 27, 453-468.

Zhao, L. and Yang Y. (2015), "Enhanced aeroelastic energy harvesting with a beam stiffener". Smart Materials and Structures, 24, 032001.

Zhao, L. and Y. Yang (2015), "Analytical solutions for galloping-based piezoelectric energy harvesters with various interfacing circuits". Smart Materials and Structures, 24, 075023. 
This article has been published as "Zhao, L., \& Yang, Y. (2018). An impact-based broadband aeroelastic energy harvester for concurrent wind and base vibration energy harvesting. Applied Energy, 212, 233243" with doi: 10.1016/j.apenergy.2017.12.042

Zhao, L. and Yang Y. (2017), "Comparison of four electrical interfacing circuits in wind energy harvesting". Sensors and Actuators A: Physical, 261, 117-129.

Zhao, L. and Yang Y. (2017), "On the modeling methods of small-scale piezoelectric wind energy harvesting". Smart Structures and Systems, 19, 67-90.

Zhou, S., Cao J., Erturk A. and Lin J. (2013), "Enhanced broadband piezoelectric energy harvesting using rotatable magnets". Applied Physics Letters, 102, 173901.

Zhou, S., Cao J., Inman D.J., Lin J., Liu S. and Wang Z. (2014), "Broadband tristable energy harvester: modeling and experiment verification". Applied Energy, 133, 33-39.

Zhou, W., Penamalli G.R. and Zuo L. (2011), "An efficient vibration energy harvester with a multi-mode dynamic magnifier". Smart Materials and Structures, 21, 015014.

Zhu, G., Peng, B., Chen, J., Jing, Q. and Wang, Z. L. (2015), "Triboelectric nanogenerators as a new energy technology: From fundamentals, devices, to applications”. Nano Energy, 14, 126-138. 